\title{
Impact Activation Improves Rapid Button Pressing
}

\author{
Sunjun Kim ${ }^{* 1,2}$, Byungjoo Lee ${ }^{* 2}$, and Antti Oulasvirta ${ }^{1}$ \\ ${ }^{1}$ Aalto University, ${ }^{2}$ Graduate School of Culture Technology, KAIST \\ \{ sunjun.kim, antti.oulasvirta\}@aalto.fi, byungjoo.lee@kaist.ac.kr
}

\begin{abstract}
The activation point of a button is defined as the depth at which it invokes a make signal. Regular buttons are activated during the downward stroke, which occurs within the first 20 $\mathrm{ms}$ of a press. The remaining portion, which can be as long as $80 \mathrm{~ms}$, has not been examined for button activation for reason of mechanical limitations. The paper presents a technique and empirical evidence for an activation technique called Impact Activation, where the button is activated at its maximal impact point. We argue that this technique is advantageous particularly in rapid, repetitive button pressing, which is common in gaming and music applications. We report on a study of rapid button pressing, wherein users' timing accuracy improved significantly with use of Impact Activation. The technique can be implemented for modern push-buttons and capacitive sensors that generate a continuous signal.
\end{abstract}

\section{ACM Classification Keywords}

H.5.2. Information Interfaces and Presentation (e.g. HCI): User Interfaces; Input devices and strategies.

\section{Author Keywords}

Impact activation; button design; activation point; temporal pointing; rapid tapping

\section{INTRODUCTION}

A button - more specifically, an electronic push-button - is a transducer that invokes a discrete signal from continuous movement. Modern buttons are typically engineered for comfort, speed, and reliable activation. This paper looks at an important goal in button design: the generation of the activation signal at an accurate moment, aligning as much as possible with the user's intended timing. This property is essential in, for example, music, gaming, and typing, or generally in any activity that requires temporal control of keystroking. Because buttons are ubiquitous, improvements in their performance may affect a large number of applications.

This paper contributes to the engineering of activation points, defined as distance at which the button generates the signal. We investigate an activation technique for improving temporal

Permission to make digital or hard copies of part or all of this work for personal or classroom use is granted without fee provided that copies are not made or distributed for profit or commercial advantage and that copies bear this notice and the full citation on the first page. Copyrights for third-party components of this work must be honored. For all other uses, contact the owner/author(s).

CHI 2018 April 21-26, 2018, Montreal, QC, Canada

(C) 2018 Copyright held by the owner/author(s).

ACM ISBN 978-1-4503-5620-6/18/04

DOI: https: //doi .org/10.1145/3173574. 3174145

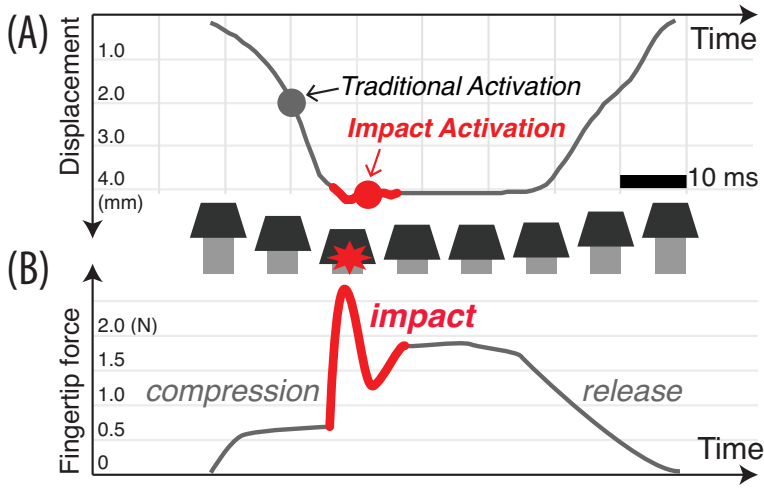

Figure 1. (A) Button displacement and (B) fingertip force curves for button pressing [18]. Traditional design activates the button during downward travel, whereas Impact Activation triggers near the point of maximum impact.

accuracy of rapid button pressing. Regular buttons invoke a make signal during downstroke, generally at $50 \%-70 \%$ of the downward travel. The activation point at downstroke is often placed at the beginning of the button-pressing duration (<20 ms). This design has been favored for its mechanical simplicity and reliability. However, the total duration of a button press is around $100 \mathrm{~ms}$ [9]. Prior to this work, there was little investigation of the remaining portion of a press.

We define an activation principle called Impact Activation (IA) and assess it empirically in a rapid tapping task. In IA, the signal is invoked at the maximal impact point during a button press (Figure 1). This point can be physically defined and implemented in sensors that provide continuous signal. At this impact point, maximum energy is transferred to the contact surface. It is also the moment when fingertip mechanoreceptors produce peak sensation. According to signal detection theory [31], stronger sensation provides more reliable evidence for event detection. It is also known that timing estimates improve when sensory amplitude increases and variance of sensory signal decreases [4]. Our hypothesis is that, thanks to better alignment of the real activation point and the consequent sensation, users can better calibrate their timing [1]

In the rest of the paper, we first define the technique and briefly review related literature. We then describe the implementation of IA with a physical button and a touch button. Finally, we compare IA with existing common button activation methods by presenting a fast tapping study. For the study, we designed a

\footnotetext{
*SK and BL contributed equally to this work.
} 
touch button and a keyboard button apparatus that can monitor the entire stream of button pressing and implemented IA for these. We expected IA to be advantageous at near-the-limit pace, as in cases of 2-5 button presses per second. In the experiment, IA significantly improved performance: we observed an increase of $94.9 \%$ on success rate with a physical button and an increase of $37.0 \%$ with a touch button.

\section{RELATED WORK}

We first discuss general features of a button, showing that design of activation points has gained little attention in prior work. We then review findings from four temporal tasks: fast tapping, synchronization, temporal pointing, and timing estimation. They suggest opportunities to improve performance in fast tapping by changing the point of activation.

\section{Design Space: Activation Point and Other Parameters}

Button design is known to affect users' peak force, tapping rate, comfort level, and muscle activity $[17,19]$. The defining parameters of a button's response are travel depth, spring stiffness, and position of activation point. Force-displacement (FD) curve of a button represents the response characteristics as affected by stiffness and mechanical design of the switch. "Snappy" buttons exhibit an "roller-coaster-like" FD curve (Figure 3). Snap force and snap ratio are determined by peak force and the depth of a valley. The activation point is commonly set within the valley area. However, the activation point is rarely, if ever, set at maximum travel, because of potential for chattering (an oscillating connect-break issue). For this work, we kept all other parameters constant and manipulated only the design of the activation point.

\section{Fast Tapping}

Fast tapping - repeated button pressing with a sub-500-ms interval - is common in games and music applications. Studies have shown that the shorter the interval, the less time users have to cognitively prepare for the next tap [33]. This suggests that any asynchrony between perceived and real moment of activation may be amplified at high rates. Studies have found that user performance can vary up to $150 \%$, depending on the input device used in playing the given game $[3,37,36]$. Our hypothesis is that different devices and input methods cause different levels of asynchrony, which may be alleviated with impact activation. A button design that improves timing accuracy might also aid in fast tapping.

\section{Synchronization}

So-called sensorimotor synchronization tasks require synchronizing tapping with a recurring reference signal (e.g., metronome) [21, 25]. Common indices of performance are mean asynchrony between stimulus onset and tap and the standard deviation of asynchrony $\left(S D_{a s y}\right)$ of taps. It has been found that people's tapping tends to precede the reference, an effect called negative mean asynchrony (NMA). Factors that affect NMA and $S D_{a s y}$ are inter-onset interval (IOI), stimulus modality, and stimulus duration. It is known that NMA increases (gets worse) with IOI $[22,38] . S D_{a s y}$ too increases with IOI [23]. Musicians exhibit less NMA than non-musicians [11]. Auditory modality appears to yield better temporal resolution (up to $100 \mathrm{~ms}$ ) than visual modality (up to $400 \mathrm{~ms}$ ) [10, 20, 24]. The highest achievable tapping rate is limited by endeffector movement, at around 5-7/s (IOI 150-200 ms) [8, 17, 27]. We believe that button design may affect NMA. For example, musicians can decrease NMA when using their own musical instrument - albeit by 2 ms only. Kaaresoja et al. [7] found that too early audio and visual feedback $(<20 \mathrm{~ms})$ may adversely affect simultaneity perception. From those phenomena, we hypothesize that the compression (downstroke) phase may not be the optimal timing for button activation.

\section{Temporal Pointing}

Temporal pointing refers to all tasks that must perform discrete inputs within a short time window [13]. Previous work explored the effect of activation point $[12,13]$. A technique called TouchMax [13], which can be considered an instance of IA for touchscreens, resulted in a significant reduction in error rate, of about $5 \%$ of the baseline result. However, the study used slow-rate tapping, and the latency of the input device was not controlled. We have explored the potential of IA in a latency-controlled system and a fast temporal pointing task that is more common in games and music applications.

\section{Timing Estimates}

In tasks that require accurate timing, users must form an integrated percept (e.g., "when finger touched button") that they can use to calibrate their responses. In button pressing, the deformation of the fingertip and proprioceptive sense of finger movement are relevant feedback signals for this purpose. A fingertip force profile measured by Rempel et al. [18] during button pressing showed that the peak force is highest at the impact point when the button travels all the way to the bottom. With a zero-travel touch button in a tapping study [5], participants pressed a button with 5-7 $\mathrm{N}$ of force, with some ramp-up time needed to reach its peak force. Tactile sensors of a fingertip are constantly stimulated during a button pressing. It is theorized that people integrate noisy sensory cues [4] into one perceptual point, called the p-center [14]. Because the mechanoreceptive signal best corresponds to the moment of impact activation, we hypothesize that users use that as the basis for their p-center.

\section{RATIONALE AND IMPLEMENTATION FOR IA}

Unlike traditional button activation methods, IA triggers a button at the point of maximum impact (see Figure 1). Figure 1b shows a fingertip force profile measured during a button press [18] from a snap-feeling button with $0.6 \mathrm{~N}$ activation force. The figure shows three main phases of a press: compression, impact, and release. The compression and release phase correspond to downward and upward movement, respectively. In the impact phase, a fingertip bumps to the button's bottom and decelerates rapidly. This yields the strongest sensation.

\section{Motor Control Rationale}

Our rationale stems from the physical fact that maximum impact results in peak force on the fingertip throughout the press. At this moment, maximum energy is transferred to the mechanoreceptors of the fingertip, which is the most salient moment in time for the perceptual system [14]. In contrast, 


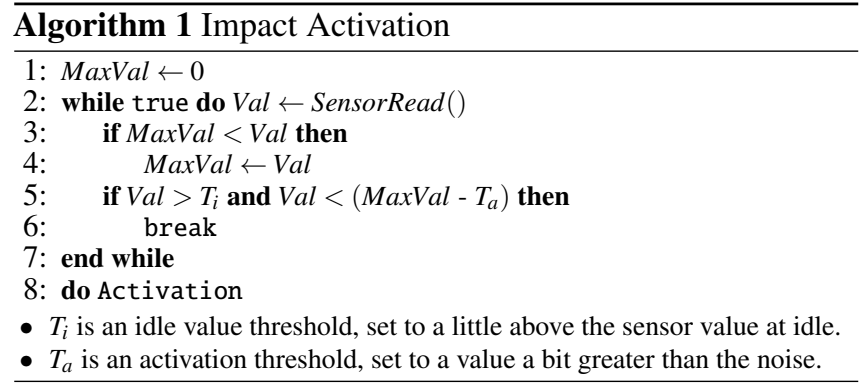

traditional buttons set the activation point only within the compression and release phase. This choice is made for practical reasons, such as double-actuation prevention and shock absorption. However, during those two phases, tactile sensation is not as brief or intense as at impact point. This may result in a mismatch between the perceived and real button activation.

Our hypotheses for the benefit of IA in fast button-pressing are threefold. First, as said, in IA the button is activated at the point corresponding with the strongest mechanoreceptive stimulation. Second, we hypothesize that the perceived timing of the finger pressing down will be better coaligned in IA, because of this reason. Consequently, users should be better able to calibrate their responses with the displayed stimulus. A recent neuromechanical model of button pressing predicts this effect [16]. Third, this effect should be more pronounced in fast repetitive tapping. In fast tapping, motor noise dominates rather than temporal noise [34], and because of the short IOI, there is not enough time for the user to correct motion. This means that the positive effect of IA would diminish at slower rates of tapping, because the temporal noise masks the advantage of IA.

\section{Method for Detecting Impact Point}

Impact occurs when a button hits its mechanical limit. To detect this, we developed an algorithm that finds the first local maximum signal during a button press. The algorithm keeps updating the maximum sensor value and activates the button if the sensor value starts to decrease (see Algorithm 1 and Figure 1a for details). For a noisy signal, $T_{a}$ may be increased, or smoothing applied before a pass through the algorithm.

Implementing the algorithm with a physical button necessitates tracking the displacement continuously throughout a button press. Readily available technologies are capacity sensing (Topre Switch [15]), optical reflection (Adomax Flaretech), and a magnetic Hall sensor (Mayle RAFI RS 76C). Initially, their purposes are to overcome reliability issues of a metal contact (oxidization, chattering, noise, etc.). Recently, some keyboard-makers have introduced an activation-point adjustment feature (e.g., Wooting One or the Topre RealForce RGB) with those switches. However, their activation point still can be set only to within a downstroke.

We applied the algorithm to a physical button and a touch button. For the physical button, we used a Hall-effect sensor to monitor the displacement. For the touch button, we used a capacitance sensor to approximate the fingertip force [2]. Alternatively, a force sensor may be usable.

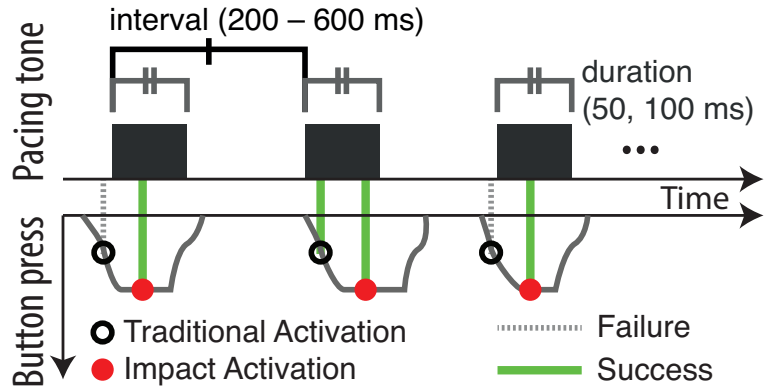

Figure 2. Illustration of the experimental task: temporal pointing. To succeed, a user must activate the button while a pacing tone is active (green lines); otherwise, the user fails (gray lines).

\section{METHOD}

The goal in the experiment was to assess the effect of IA relative to the regular activation method in terms of temporal accuracy for both physical and touch buttons. We used a version of the temporal pointing task [13] (Figure 2). This task reproduces a common requirement in games and music, where button activation must occur within some time window. Auditory pacing tones were played with regular interval and duration. We chose the audio channel since it showed better temporal synchronization performance $(10 \mathrm{~Hz})[20,24]$ than visual stimuli $(2.5 \mathrm{~Hz})$. Participants were asked to activate a button during the tone. Therefore, a successful trial meant detection of button activation during the tone, and failure otherwise. To inform the user of success/failure, brief visual feedback (a flashing LED for $50 \mathrm{~ms}$ at the moment of activation) was given. To rule out confusion between tone and feedback signals, audio feedback was avoided.

We chose a tapping rate with intervals of 200 to $600 \mathrm{~ms}$ (or 100-300 beats per minute), which are common tempos found in music and games. There were three reasons for this choice. First, synchronizing with a high rate, close to the limit of one's ability (around $200 \mathrm{~ms}[17,27]$ ), is more challenging and yields a more sensitive performance metric. Second, it can reduce strategy variation. We hypothesized that participants perform the task instinctively at higher rates, whereas with lower rates they may behave strategically. Third, 100 trials takes only 40 seconds, on average, which allowed us to explore more experimental conditions.

\section{Participants}

In total, 32 participants (18 male, 14 female; on average, 26.0 years old; $S D=5.0$ ) were recruited from a local university in Helsinki, Finland. Twenty-one had experience of musical training (at least basic level, avg. 7.2 years, $S D=5.8$ ). Three were left-handed. Most used the index finger of the dominant hand for the study. One used the thumb and three used the middle finger. They were compensated with a movie ticket.

\section{Experiment Design}

The experiment followed a $2 \times 2$ within-subject factorial design. There were two independent factors: button type and activation method, each with two levels. Within a condition, combinations of five tone intervals and two tone durations were examined. Each combination consisted of 100 trials. 


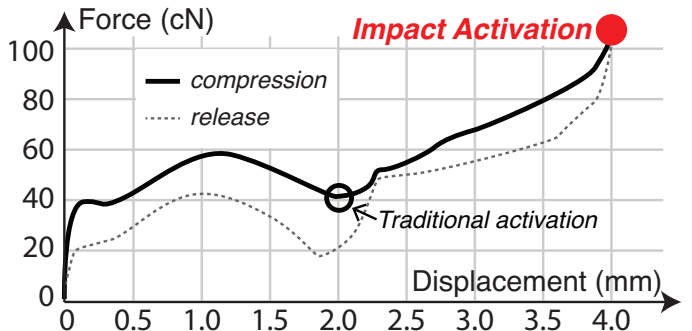

Figure 3. Force-displacement profile of the PHYSICAL button, measured from the Cherry MX MX1AC1NN switch used in the experiment. Points of activations (traditional and impact) are also illustrated.

In summary, the design was:

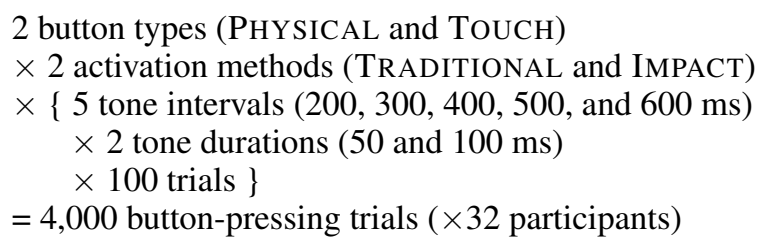

Using a Balanced Latin Square [32], we counterbalanced the presentation order of four conditions: PHYSICALTraditional, PhysiCAl-IMPaCt, TOUCh-TRAditional, and TOUCH-IMPACT. There were four training blocks and ten test blocks ( 5 intervals $\times 2$ durations) within a condition. The training blocks consisted of 400 (interval)/100(duration), $400 / 75$, and two 200/50 trials. Presentation order of the test blocks was randomized.

\section{Apparatus}

To exclude external disturbances, a custom-designed lowlatency instrument was used. An Arduino Leonardo board processed all the signal generation and acquisition internally, to ensure minimal latency [7, 26]. For logging, data frames were transmitted to a PC with an internally-generated timestamp attached; thereby, communication latency could be avoided. The measured sampling rate of the instrument was 542 frames per second, which ensures a worst-case latency of $1.85 \mathrm{~ms}$.

The PHYSICAL condition used a mechanical key switch (Cherry MX MX1AC1NN) that snaps at 1-2 mm displacement with $60 \mathrm{cN}$ force (snap ratio $=33 \%$ ) and hits its maximal travel with $100 \mathrm{cN}$ force at $4 \mathrm{~mm}$ (see Figure 3). A linear Halleffect sensor (SS49E) attached to pin AO monitored the button displacement with a small neodymium magnet attached to a keycap (Figure 4). Because the Hall sensor signal is non-linear with respect to displacement, we calibrated the sensor with a precision moving platform to press a button. We made a table of sensor values at $0.1 \mathrm{~mm}$ resolution and interpolated them to map the raw signal to a metric-unit displacement value.

The TOUCH condition used a touch sensor (Adafruit MPR121 module $\left.{ }^{1}\right)$. A copper plate $(20 \times 40 \mathrm{~mm})$ was attached to pin 0 of it. All other input pins were disabled for shorter scan time. We obtained the capacitance value by subtracting between two

\footnotetext{
${ }^{1}$ See https://www . adafruit. com/product/1982.
}

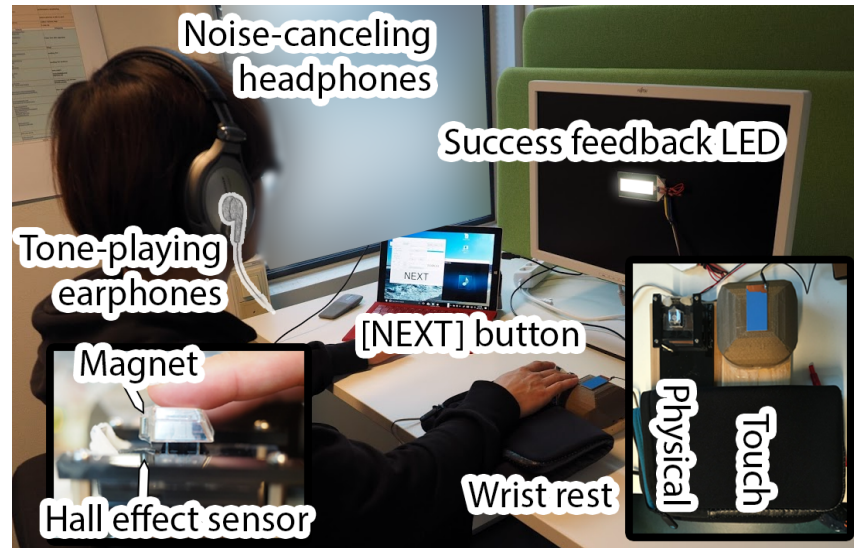

Figure 4. Experiment apparatus and setup.

raw values (baselineData() and filteredData() functions), using the dedicated Adafruit MPR121 library.

The PHYSICAL and Touch buttons were connected to the Arduino board. It monitored the button signals and activated the button with a given activation-method setting. With TRADITIONAL activation, the PHYSICAL button was activated at $2 \mathrm{~mm}$ displacement during downstroke (as its datasheet showed) and the TOUCH button was activated when a signal exceeded 10 (the default threshold is 12). With IMPACT activation, Algorithm 1 was applied with $T_{i}=2 \mathrm{~mm} / 20$ and $T_{a}=0.02$ $\mathrm{mm} / 1$, for PHYSICAL and TOUCH, respectively.

The same Arduino board generated the audio tone and visual LED feedback. The tone was generated via PWM output of pin D5 $(488 \mathrm{~Hz}, 50 \%$ duty). A pair of earphones was connected through a volume-adjusting linear potentiometer. The tone was played for the given duration (50 or $100 \mathrm{~ms}$ ) and interval (200-600 ms). If the board detected a button activation during the tone, it flashed the feedback LED for $50 \mathrm{~ms}$ immediately.

\section{Procedure}

Participants were welcomed and read the experiment consent form. After agreement, they sat on a chair and were asked to adjust the chair height to be comfortable for button pressing. We changed the chair position on the basis of their dominant hand. Participants were asked to fix their wrist to the rest. The feedback LED was located at eye level and 1 meter away from the participant. The experimenter briefly explained the task. Participants were asked to succeed in as many trials as possible, and to try to calibrate their button-pressing behavior for that. They were blinded to the activation methods. They wore a pair of earphones for pacing-tone stimuli and wore noise-canceling headphones (Sennheiser PXC-450) on top of that, which played pink noise during the experiment. The noise volume was adjusted to block the button-pressing noise, and the tone volume was adjusted to be comfortable and clearly heard by participants.

During the training block, participants calibrated their buttonpressing behavior to maximize the number of successful trials. They were able to take a short break between blocks and pressed the "next" button to start the next block. At the beginning of a block, they heard the tone for a while to get a 


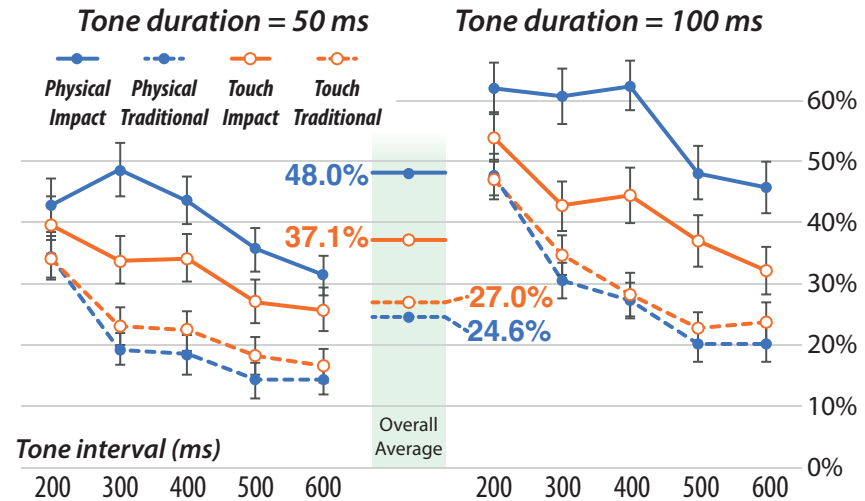

Figure 5. Success rate (error bars show standard error). Higher is better. On average, IMPACT could improve success rate in the task: for the PHYSICAL button, $\mathbf{2 4 . 6} \% \rightarrow \mathbf{4 8 . 0} \%$ and for the TOUCH button, $\mathbf{2 7 . 0 \%} \rightarrow \mathbf{3 7 . 1} \%$.

rhythm first, then started button pressing. Each participant performed 100 button-pressing trials for one block. After one condition ended, the experimenter set the next condition and repeated the 14 blocks. When four conditions were completed, we collected participants' demographic data and finished the experiment. The whole procedure took about an hour.

\section{RESULTS}

Each participant produced 40 blocks of data (10 blocks $\times$ 4 conditions). The first 20 trials in a block were discarded as practice trials, and the remaining 80 were included in the analysis. Because of experimental-program error, three blocks from P2 were not recorded. Therefore, 1277 blocks $(32 \times 40-$ 3 ) were analyzed in total. We performed repeated-measures ANOVA for statistical testing. The success rate was calculated as $\#$ \# of successful trials trials $(=80)$.

\section{Normality of Distributions}

We tested the normality of button activation timing distribution with one-sample Kolmogorov-Smirnov testing. Of 1,277 blocks, $81.6 \%$ (or 1,042 blocks) followed the normality assumption $(\alpha=.05)$. Therefore, most of the data could be abstracted into a normal distribution with mean asynchrony and standard deviation.

\section{Success Rate}

For success rates (see Figure 5), the difference between TRADITIONAL and IMPACT was large and statistically significant. When compared to TRADITIONAL, the overall success rate of IMPACT increased by $94.9 \%(23.4 \%$ p) for PHYSICAL and $37.0 \%$ (10.0\%p) for TOUCH buttons.

Two-way RM-ANOVA showed that button type $\left(F_{(1,31)}=7.33\right.$, $\left.p=.011, \quad \eta_{\text {partial }}^{2}=.19\right)$, activation method $\left(F_{(1,31)}=52.08\right.$, $\left.p<.001, \eta_{\text {partial }}^{2}=.63\right)$, and their interaction $\left(F_{(1,31)}=19.41\right.$, $p<.001, \eta_{\text {partial }}^{2}=.39$ ) had a significant effect on success rate. Since they showed an interaction effect, we separately compared IMPACT and TRADITIONAL within each button type, using a paired-samples $t$-test. Significant differences between activation methods were found for both button types $\left(t_{(31)}=7.30\right.$ within PHYSICAL and $t_{(31)}=4.43$ within TOUCH, all $\left.p<.001\right)$.

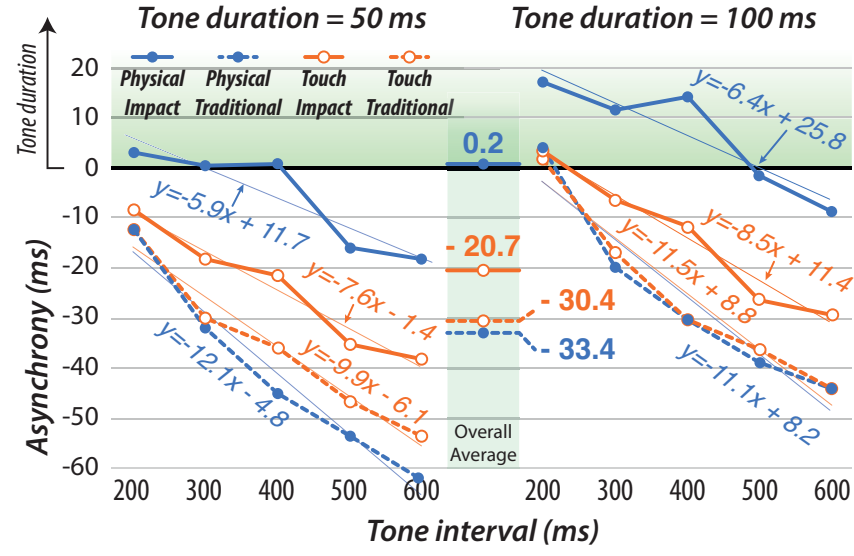

Figure 6. Mean asynchrony between stimulus tone onset $(=0 \mathrm{~ms})$ and button activation time, Closer to zero is better.

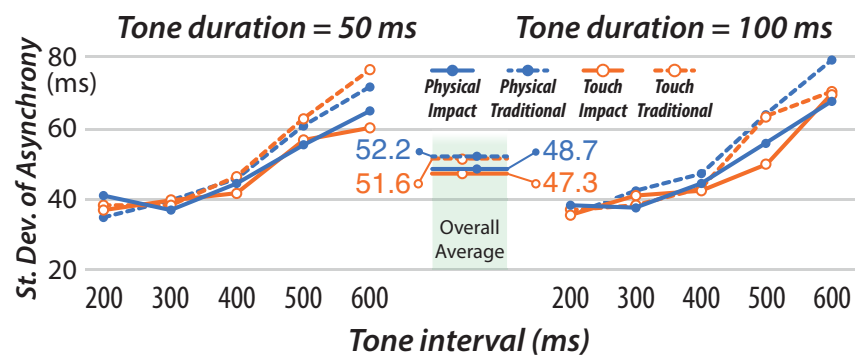

Figure 7. Standard deviation of the amount of asynchrony $\left(S D_{a s y}\right)$.

IMPACT had significantly better performance for both button types. PHYSICAL and TOUCH exhibited almost identical performance with the TRADITIONAL activation method. With IMPACT activation, PHYSICAL conditions were superior to TOUCH conditions.

\section{Asynchrony}

Compared to TRADITIONAL, IMPACT showed less asynchrony between the tone onset and the button activation time (Figure 6). Asynchrony closer to zero is considered better. The NMA increased with tone interval (consistent with [22, 38]). The shorter duration $(50 \mathrm{~ms})$ condition exhibited more NMA than $100 \mathrm{~ms}$ duration ones (consistent with [13, 29]).

Two-way RM-ANOVA showed that button type $\left(F_{(1,31)}=14.21\right.$, $\left.p=.001, \eta_{\text {partial }}^{2}=.31\right)$, activation method $\left(F_{(1,31)}=43.16\right.$, $\left.p<.001, \eta_{\text {partial }}^{2}=.58\right)$, and their interaction $\left(F_{(1,31)}=18.65\right.$, $p<.001, \eta_{\text {partial }}^{2}=.38$ ) all displayed a significant effect on mean asynchrony. Paired-samples $t$-tests revealed a significant difference in mean asynchrony between the activation methods for both button types $\left(t_{(31)}=6.76, p<.001\right.$ within PHYSICAL and $t_{(31)}=3.29, p=.003$ within TOUCH). Linear fitting showed the slope of IMPACT activation (-6.5 to -8.5$)$ to be more gradual than that of TRADITIONAL activation (-10.2 to -12.2). In summary, IMPACT indeed exhibited better alignment with tone onset and was more robust to interval changes.

\section{Standard Deviation of Asynchrony}

Compared to TRADITIONAL, IMPACT showed a smaller $S D_{a s y}$ (see Figure 7), which represents better temporal stability. 


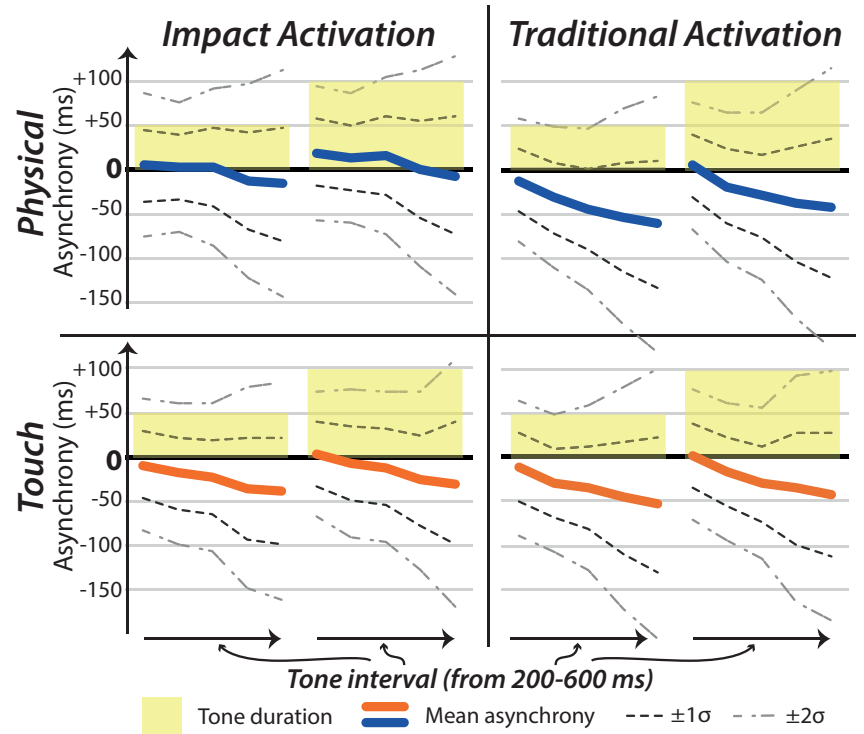

Figure 8. Illustration of button-pressing distributions (approximated to normal) with mean asynchrony (thick lines) and their deviations. Empirically, $\pm 1 \sigma$ lines and $\pm 2 \sigma$ lines indicate that $68 \%$ and $95 \%$ of data points are likely to fall within those lines. The yellow-shaded zone indicates the tone duration. More overlap with that zone means better success rates. See figures 6 and 7 for the breakdown of results.

Two-way RM-ANOVA showed that only activation method exhibited a significant effect on $S D_{a s y}\left(F_{(1,31)}=6.33, p=.02\right.$, $\left.\eta_{\text {partial }}^{2}=.17\right)$. Button type $\left(F_{(1,31)}=.249, p=.621\right)$ and the two's interaction $\left(F_{(1,31)}=.043, p=.838\right)$ did not exhibit a significant effect. On average, $S D_{\text {asy }}$ was $8.2 \%$ smaller in IMPACT activation conditions $(47.8 \mathrm{~ms})$ than TRADITIONAL $(52.0 \mathrm{~ms})$, which is in line with previous literature $[12,13]$. This means that participants could perform button pressing more stably with IMPACT. Figure 8 plots the distribution for each condition, with tone onset and duration overlaid. Dominance of the PHYSICAL-IMPACT condition is clearly visible.

\section{Musicians vs. Non-Musicians}

Musicians are known to exhibit better temporal accuracy than non-musicians [11]. We separated among participants by musical education experience and compared them. Relative to the non-musician group (11 people), the musician group (21 people) indeed exhibited better success rates, less asynchrony, and less standard deviation (see Table 1). Also, the difference in performance increment with IMPACT was pronounced. For the musicians, PHYSICAL success rate was increased by $112 \%$ and TOUCH success rate was higher by $38 \%$. In comparison, the non-musician group exhibited only a $60 \%$ increment in PHYSICAL and $34 \%$ increment in TOUCH. However, we could still observe a performance gain of IMPACT over TRADITIONAL irrespective of group, which is impressive.

\section{CONCLUSION}

For this paper, we defined and empirically investigated an activation technique called Impact Activation. A user study showed IA to be superior to the traditional button activation methods for all performance metrics in a fast tapping task: IA had higher success rates, less asynchrony, and less variance

\begin{tabular}{rr|rr|rr} 
& & \multicolumn{2}{|c|}{ Physical } & \multicolumn{2}{c}{ Touch } \\
& & Impact & Traditional & Impact & Traditional \\
\hline $\begin{array}{r}\text { Success } \\
\text { rate }\end{array}$ & N.M & $52.8 \%$ & $24.9 \%$ & $39.9 \%$ & $28.9 \%$ \\
\hline Async- & $38.9 \%$ & $24.2 \%$ & $31.7 \%$ & $23.6 \%$ \\
rony & N.M & $3.7 \mathrm{~ms}$ & $-33.0 \mathrm{~ms}$ & $-14.4 \mathrm{~ms}$ & $-26.3 \mathrm{~ms}$ \\
\hline Stdev. & M & $41.4 \mathrm{~ms}$ & $-34.1 \mathrm{~ms}$ & $28.2 \mathrm{~ms}$ & $-38.3 \mathrm{~ms}$ \\
& N.M & $62.0 \mathrm{~ms}$ & $60.4 \mathrm{~ms}$ & $58.6 \mathrm{~ms}$ & $61.3 \mathrm{~ms}$
\end{tabular}

Table 1. Performance comparison between the musician group (M) and non-musician group (N.M). The musicians exhibited better success rates, less asynchrony, and less standard deviation of asynchrony.

(see figures 6 and 7). The performance gains were greater in the physical button condition than in the touch button condition.

The benefit of IA is likely to be attributable to the stronger stimulation of the fingertip allowing the motor system to calibrate its outputs better in the fast, episodic tapping task. We hypothesize that IA is closer to users' perceived activation point than a traditional button activation point is. The activation point design choice had a much greater effect on the mean of asynchrony than on its standard deviation.

To the best of our knowledge, the design of buttons has not been approached before by exploiting findings from theories of temporal motor control. Our work may reveal a new opportunity to understand why user performance has been much worse with touchscreen devices than physical input devices. In HCI research, work on touch input has focused, for example, on the "fat finger problem" [28] and system latency $[6,35]$. Techniques such as IA that are motivated by motor control literature may complement the gains achieved in these areas. Furthermore, it could address well-known performance impairments reported for touchscreen gaming [3, 36, 37].

We see several opportunities to build on this work. First, our results are limited to a particular modality combination: audio cue with visual feedback. We believe the benefit of IA will persist with other modalities, but it may be smaller. This remains a topic for future work. Also, our experimental task was relatively short, so there may be some quickly obtained advantages to using IA, particularly for novice users. To assess the robustness of the effect, a longitudinal study should be carried out. Longitudinal research would expose possible recalibration effects [30]. Finally, our experiment tested two specific button designs. Although these represent commonly used types, we see it as worthwhile to replicate the work with other button designs.

\section{Acknowledgments}

This research was supported by the European Research Council (ERC) under the European Union's Horizon 2020 research and innovation program (grant agreement 637991) and the Basic Science Research Program through the National Research Foundation of Korea (NRF), funded by the Ministry of Science and ICT (NRF-2017R1C1B2002101). We also thank Jong-In Lee for help with the experiment and Jussi Jokinen for advice on statistical-test reporting. 


\section{REFERENCES}

1. Gisa Aschersleben. 2002. Temporal Control of Movements in Sensorimotor Synchronization. Brain and Cognition 48, 1 (2002), 66 - 79. DOI : http://dx.doi.org/10.1006/brcg.2001.1304

2. Sebastian Boring, David Ledo, Xiang 'Anthony' Chen, Nicolai Marquardt, Anthony Tang, and Saul Greenberg. 2012. The Fat Thumb: Using the Thumb's Contact Size for Single-handed Mobile Interaction. In Proceedings of the 14th International Conference on Human-computer Interaction with Mobile Devices and Services (MobileHCI '12). ACM, New York, NY, USA, 39-48. DOI :http://dx.doi .org/10.1145/2371574.2371582

3. Kimberly Chu and Chui Yin Wong. 2011. Mobile input devices for gaming experience. In 2011 International Conference on User Science and Engineering (i-USEr ). 83-88. DOI:

http://dx.doi.org/10.1109/iUSEr.2011.6150542

4. Marc O Ernst and Martin S Banks. 2002. Humans integrate visual and haptic information in a statistically optimal fashion. Nature 415, 6870 (2002), 429-433. DOI : http://dx.doi .org/10.1038/415429a

5. Curt B. Irwin and Mary E. Sesto. 2012. Performance and touch characteristics of disabled and non-disabled participants during a reciprocal tapping task using touch screen technology. Applied Ergonomics 43, 6 (2012), $1038-1043$. DOI :

http://dx.doi.org/10.1016/j. apergo.2012.03.003

6. Topi Kaaresoja, Emilia Anttila, and Eve Hoggan. 2011. The effect of tactile feedback latency in touchscreen interaction. In 2011 IEEE World Haptics Conference. 65-70. DOI : http://dx.doi .org/10.1109/WHC. 2011.5945463

7. Topi Kaaresoja, Stephen Brewster, and Vuokko Lantz. 2014. Towards the Temporally Perfect Virtual Button: Touch-Feedback Simultaneity and Perceived Quality in Mobile Touchscreen Press Interactions. ACM Trans. Appl. Percept. 11, 2, Article 9 (June 2014), 25 pages. DOI : http://dx.doi.org/10.1145/2611387

8. Steven W. Keele and Harold L. Hawkins. 1982. $>$ Explorations of Individual Differences Relevant to High Level Skill. Journal of Motor Behavior 14, 1 (1982), 3-23. DOI :

http://dx.doi.org/10.1080/00222895.1982.10735259 PMID: 15151886.

9. Sunjun Kim, Jeongmin Son, Geehyuk Lee, Hwan Kim, and Woohun Lee. 2013. TapBoard: Making a Touch Screen Keyboard More Touchable. In Proceedings of the SIGCHI Conference on Human Factors in Computing Systems (CHI '13). ACM, New York, NY, USA, 553-562. DOI : http://dx.doi.org/10.1145/2470654.2470733

10. Paul A Kolers and Joan M Brewster. 1985. Rhythms and responses. Journal of Experimental Psychology: Human Perception and Performance 11, 2 (1985), 150-167. DOI : http://dx.doi.org/10.1037/h0090450
11. Vanessa Krause, Bettina Pollok, and Alfons Schnitzler. 2010. Perception in action: The impact of sensory information on sensorimotor synchronization in musicians and non-musicians. Acta Psychologica 133, 1 (2010), 28 - 37. DOI :

http://dx. doi .org/10.1016/j. actpsy. 2009.08.003

12. Byungjoo Lee, Qiao Deng, Eve Hoggan, and Antti Oulasvirta. 2017. Boxer: A Multimodal Collision Technique for Virtual Objects. In Proceedings of the 19th ACM International Conference on Multimodal Interaction (ICMI 2017). ACM, New York, NY, USA, 252-260. DOI : http://dx.doi.org/10.1145/3136755.3136761

13. Byungjoo Lee and Antti Oulasvirta. 2016. Modelling Error Rates in Temporal Pointing. In Proceedings of the 2016 CHI Conference on Human Factors in Computing Systems (CHI'16). ACM, New York, NY, USA, 1857-1868. DOI : http://dx.doi.org/10.1145/2858036.2858143

14. John Morton, Steve Marcus, and Clive Frankish. 1976. Perceptual centers (P-centers). Psychological Review 83, 5 (1976), 405. DOI :

http://dx.doi.org/10.1037/0033-295x.83.5.405

15. Takao Nagashima. 1986. Keyboard switch. (April 22 1986). US Patent $4,584,444$.

16. Antti Oulasvirta, Sunjun Kim, and Byungjoo Lee. 2018. Neuromechanics of a Button Press. In Proceedings of the 36th Annual ACM Conference on Human Factors in Computing Systems (CHI'18). ACM Press. DOI : http://dx.doi.org/10.1145/3173574.3174082

17. Robert G. Radwin and One-Jang Jeng. 1997. Activation Force and Travel effects on Overexertion in Repetitive Key Tapping. Human Factors 39, 1 (1997), 130-140. DOI: http://dx.doi.org/10.1518/001872097778940605 PMID: 9302885.

18. David Rempel, Jack Dennerlein, C.D. Mote, and Thomas Armstrong. 1994. A method of measuring fingertip loading during keyboard use. Journal of Biomechanics 27, 8 (1994), 1101 - 1104. DOI: http://dx.doi .org/10.1016/0021-9290(94)90227-5

19. David Rempel, Elaine Serina, Edward Klinenberg, Bernard J Martin, Thomas J Armstrong, James A Foulke, and Sivakumaran Natarajan. 1997. The effect of keyboard keyswitch make force on applied force and finger flexor muscle activity. Ergonomics 40, 8 (1997), 800-808.

20. Bruno H. Repp. 2003. Rate Limits in Sensorimotor Synchronization With Auditory and Visual Sequences: The Synchronization Threshold and the Benefits and Costs of Interval Subdivision. Journal of Motor Behavior 35, 4 (2003), 355-370. DOI:

http://dx.doi.org/10.1080/00222890309603156 PMID: 14607773.

21. Bruno H. Repp. 2005. Sensorimotor synchronization: A review of the tapping literature. Psychonomic Bulletin \& Review 12, 6 (01 Dec 2005), 969-992. DOI:

http://dx.doi.org/10.3758/BF03206433 
22. Bruno H. Repp. 2008. Multiple temporal references in sensorimotor synchronization with metrical auditory sequences. Psychological Research 72, 1 (01 Jan 2008), 79-98. DOI :

http://dx.doi.org/10.1007/s00426-006-0067-1

23. Bruno H Repp. 2012. Comments on âĂIJThe effects of stimulus rate and tapping rate on tapping performanceâĂ by Zendel, Ross, and Fujioka (2011). Music Perception: An Interdisciplinary Journal 29, 4 (2012), 447-448.

24. Bruno H Repp and Amandine Penel. 2002. Auditory dominance in temporal processing: new evidence from synchronization with simultaneous visual and auditory sequences. Journal of Experimental Psychology-Human Perception and Performance 28, 5 (2002), 1085-1099. DOI : http://dx.doi.org/10.1037/0096-1523.28.5.1085

25. Bruno H. Repp and Yi-Huang Su. 2013. Sensorimotor synchronization: A review of recent research (2006-2012). Psychonomic Bulletin \& Review 20, 3 (01 Jun 2013), 403-452. DOI : http://dx.doi.org/10.3758/s13423-012-0371-2

26. Benjamin G. Schultz and Floris T. van Vugt. 2016. Tap Arduino: An Arduino microcontroller for low-latency auditory feedback in sensorimotor synchronization experiments. Behavior Research Methods 48, 4 (01 Dec 2016), 1591-1607. DOI :

http://dx.doi.org/10.3758/s13428-015-0671-3

27. Martine Turgeon, Alan M Wing, and Lawrence W Taylor. 2011. Timing and aging: Slowing of fastest regular tapping rate with preserved timing error detection and correction. Psychology and Aging 26, 1 (2011), 150-161. DOI : http://dx.doi .org/doi/10.1037/a0020606

28. Daniel Vogel and Patrick Baudisch. 2007. Shift: A Technique for Operating Pen-based Interfaces Using Touch. In Proceedings of the SIGCHI Conference on Human Factors in Computing Systems (CHI '07). ACM, New York, NY, USA, 657-666. DOI : http://dx.doi.org/10.1145/1240624.1240727

29. Piet G. Vos, JiÅŹ̃̃̄ Mates, and Noud W. van Kruysbergen. 1995. The Perceptual Centre of a Stimulus as the Cue for Synchronization to a Metronome: Evidence from Asynchronies. The Quarterly Journal of Experimental Psychology Section A 48, 4 (1995), 1024-1040. DOI :

http://dx.doi.org/10.1080/14640749508401427
30. Jean Vroomen, Mirjam Keetels, Beatrice de Gelder, and Paul Bertelson. 2004. Recalibration of temporal order perception by exposure to audio-visual asynchrony. Cognitive Brain Research 22, 1 (2004), 32 - 35. DOI : http://dx.doi.org/10.1016/j. cogbrainres.2004.07.003

31. Christopher D Wickens, Justin G Hollands, Simon Banbury, and Raja Parasuraman. 2015. Engineering psychology \& human performance. Psychology Press.

32. EJ Williams. 1949. Experimental designs balanced for the estimation of residual effects of treatments. Australian Journal of Chemistry 2, 2 (1949), 149-168. DOI : http://dx. doi .org/10.1071/CH9490149

33. Alan M. Wing and A. B. Kristofferson. 1973a. Response delays and the timing of discrete motor responses. Perception \& Psychophysics 14, 1 (01 Feb 1973), 5-12. DOI : http://dx.doi .org/10.3758/BF03198607

34. Alan M. Wing and A. B. Kristofferson. 1973b. The timing of interresponse intervals. Perception \& Psychophysics 13, 3 (01 Oct 1973), 455-460. DOI : http://dx.doi.org/10.3758/BF03205802

35. Haijun Xia, Ricardo Jota, Benjamin McCanny, Zhe Yu, Clifton Forlines, Karan Singh, and Daniel Wigdor. 2014. Zero-latency Tapping: Using Hover Information to Predict Touch Locations and Eliminate Touchdown Latency. In Proceedings of the 27th Annual ACM Symposium on User Interface Software and Technology (UIST '14). ACM, New York, NY, USA, 205-214. DOI : http://dx.doi.org/10.1145/2642918.2647348

36. Loutfouz Zaman and I Scott MacKenzie. 2013. Evaluation of nano-stick, foam buttons, and other input methods for gameplay on touchscreen phones. In International Conference on Multimedia and Human-Computer Interaction-MHCI 2013. 69-1.

37. Loutfouz Zaman, Daniel Natapov, and Robert J. Teather. 2010. Touchscreens vs. Traditional Controllers in Handheld Gaming. In Proceedings of the International Academic Conference on the Future of Game Design and Technology (Futureplay '10). ACM, New York, NY, USA, 183-190. DOI :

http://dx.doi.org/10.1145/1920778.1920804

38. Benjamin Rich Zendel, Bernhard Ross, and Takako Fujioka. 2011. The Effects of Stimulus Rate and Tapping Rate on Tapping Performance. Music Perception: An Interdisciplinary Journal 29, 1 (2011), 65-78. DOI : http://dx.doi.org/10.1525/mp.2011.29.1.65 